\title{
Finite-size effects on the magnetoelectric coupling in a ferroelectric /ferromagnetic structure revealed by ferromagnetic resonance
}

\author{
A. Sukhov ${ }^{1,2}$ a, P.P. Horley ${ }^{2}$, C.-L. Jia ${ }^{3}$, L. Chotorlishvili ${ }^{1}$, and J. Berakdar ${ }^{1}$ \\ ${ }^{1}$ Institut für Physik, Martin-Lüther-Universität Halle-Wittenberg, 06120 Halle (Saale), Germany \\ ${ }^{2}$ Centro de Investigación en Materiales Avanzados (CIMAV S.C.), Chihuahua/Monterrey, \\ 31109 Chihuahua, Mexico \\ ${ }^{3}$ Key Laboratory for Magnetism and Magnetic Materials of the Ministry of Education, \\ Lanzhou University, 730000 Lanzhou, China
}

\begin{abstract}
For a composite multiferroic chain corresponding to a $\mathrm{BaTiO}_{3} / \mathrm{Fe}$-interface we report theoretical calculations of the spectra of ferromagnetic resonance relying on the solution of the coupled LandauKhalatnikov equation for the FE polarization and the Landau-Lifshitz-Gilbert equation for the magnetization motion. We focus on the role of the system size. Additionally, we find that although the magnetoelectric coupling strength remains linear upon the electric field variation, its dependence on the thickness of the ferromagnet is not linear.
\end{abstract}

\section{Introduction}

Interest to functionally new materials - multiferroics (MFs) [1-5] is currently driven by their potential applications in spintronics and sensorics with low-level energy consumption and due to their environmentally friendly characteristics. Special attention is focused on composite multiferroic materials [6] - where more than one ferroic phase is combined, since the properties of their bulk components are well known for many materials. In addition, coupling between the ferroic phases is limited to interfaces making them simpler for both chemical and physical characterization.

It is obvious that the crucial role in controlling the properties of composite MF materials is played by the coupling between each ferroic phases. To be specific, in our study we consider a junction of $\mathrm{BaTiO}_{3} / \mathrm{Fe}-$ the composite for which even at room temperature high and stable magnetoelectric (ME) coupling [7] between ferroelectric (FE) and ferromagnetic (FM) phases was predicted [8-10] and demonstrated experimentally [11, 12]. The microscopic understanding of the ME coupling in MF composites is still under debates. Therefore, it is important to explore potential methods for measuring the ME coupling.

One of the means to handle the problem of how to access the ME coupling is to measure the magnetic hysteresis as a function of an external magnetic field. Along this way the effect is expected to be similar to the exchange bias (EB) $[13,14]$ primarily known for the interfaces of ferro- coupled with antiferromagnets
(AFMs). Typical signature of EB experiments is a horizontal shift of the hysteresis curve, caused by the interfacial FM/AFM-coupling, or the unidirectional anisotropy. Assuming that FE barium titanate is coupled to FM iron, we might expect a similar effect of shifting of the magnetic hysteresis. Since, however, the predicted ME coupling is lower than the EB anisotropy originating from the exchange interaction and also due a limited volume of the $\mathrm{ME}$ interaction, the $\mathrm{EB}$ in multiferroics might be too low.

Alternatively, another experimental technique ferromagnetic resonance (FMR) [15-17] can be employed for measuring the ME coupling because of its high magnetic sensitivity.

In our previous study [18] we have already demonstrated theoretically that the FMR technique is capable of measuring the ME-coupling strength for the junction $\mathrm{BaTiO}_{3} / \mathrm{Fe}$ and for the case of linear $\mathrm{ME}$ coupling. Here we focus on the issue of finite size of the composite and its impact on the subsequent results.

\section{Theoretical model}

The geometry of the $\mathrm{BaTiO}_{3} / \mathrm{Fe}$-junction including the explicit mutual alignment of applied magnetic fields is given in Ref. [18].

Our theoretical approach relies on the classical treatment of the discretized FE polarization of barium titanate considered in the coarse-grained approximation and denoted via $\mathbf{P}_{i}$. Thermodynamic free energy of the

\footnotetext{
a Corresponding author: alexander.sukhov@physik.uni-halle.de
} 
FE in the tetragonal phase includes the following terms [19]

$$
\begin{aligned}
F_{\mathrm{FE}}= & \sum_{i}\left(\frac{\alpha_{\mathrm{FE}}}{2} P_{\mathrm{zi}}^{2}+\frac{\beta_{\mathrm{FE}}}{4} P_{\mathrm{zi}}^{4}+\frac{\kappa_{\mathrm{FE}}}{2}\left(P_{z i+1}-P_{\mathrm{zi}}\right)^{2}-P_{\mathrm{zi}} E_{\mathrm{z}}\right) \\
& +F_{\mathrm{DDI}}^{\mathrm{FE}},
\end{aligned}
$$

where the terms $\alpha_{\mathrm{FE}}, \beta_{\mathrm{FE}}, \kappa_{\mathrm{FE}}$ describe the FE potential and the FE interaction between nearest neighbours, respectively [19], $E_{\mathrm{z}}$ - is the applied electric field and the term containing the FE dipole-dipole-interaction (DDI) together with the depolarizing field term are clarified in Ref. [20].

FM is also considered in a classical sense. The total free energy of the ferromagnetic iron with the coarsegrained magnetization $\mathbf{M}_{j}$ scales as

$$
\begin{aligned}
F_{\mathrm{FM}}= & \sum_{j}\left(-\frac{A}{a_{\mathrm{FM}}^{2} M_{\mathrm{S}}^{2}} \mathbf{M}_{j} \cdot \mathbf{M}_{j+1}-\frac{K_{1}}{M_{\mathrm{S}}^{2}} M_{\mathrm{zj}}^{2}-\mu_{0} \mathbf{M}_{j} \cdot \mathbf{H}_{\Sigma}(t)\right) \\
& +F_{\mathrm{DDI}}^{\mathrm{FM}},
\end{aligned}
$$

whereas $\mu_{0}$ - is the magnetic permittivity constant, $A$ - is the exchange stiffness of iron, $K_{1}-$ is its anisotropy strength and $M_{\mathrm{S}}$ is the magnetization at saturation. $\mathbf{H}_{\Sigma}(t)=H_{0} \cos \omega t \mathbf{e}_{\mathrm{y}}+H \mathbf{e}_{\mathrm{z}}$ denotes the total applied magnetic field [18].

For the junction of $\mathrm{BaTiO}_{3} / \mathrm{Fe}$ the recent ab-initio studies [8-10] suggested the ME coupling energy scaling according to $\lambda \mathbf{P}_{1} \cdot \mathbf{M}_{1}$, with $\lambda$ being the ME coupling strength.

Numerically, the MF system is evolved in time obeying the coupled Landau-Khalatnikov equation for the polarization dynamics and the Landau-Lifshitz-Gilbert equation for the magnetization motion [18]. After reaching an equilibrium state for the coupled polarization and magnetization, the FMR power absorbed by the MF chain is calculated [18]

$$
P_{\mathrm{FMR}}=-\mu_{0} a_{\mathrm{FM}}^{3} \frac{1}{N_{\mathrm{FM}}} \sum_{j} \frac{1}{N_{\mathrm{T}} T} \int_{0}^{N_{\mathrm{T}} T} \mathbf{M}_{j}(t) \cdot \frac{\partial \mathbf{H}_{\Sigma}}{\partial t} d t,
$$

where the summation runs over all magnetic sites denoted by $j$.

The conditions of resonance for a long FM chain will include not only the anisotropy field and the contribution from the ME coupling, but also the exchange energy contributions (exchange energy field $H_{\text {exch }}$ ). In this case expression (6) given in Ref. [18] reads

$$
\mu_{0} H_{\text {res }}=\frac{1}{\gamma} \omega-\frac{2 K_{1}}{M_{\mathrm{S}}^{2}} M_{\text {tot } \mathrm{z}}-\mu_{0} H_{\mathrm{exch}}+\lambda P_{\mathrm{z}} \text {. }
$$

In eq. (4) $H_{\text {res }}$ stands for the applied magnetic field corresponding to the highest absorbed power, $\gamma$ and $M_{\text {tot z }}$ describe the gyromagnetic ratio and the z-projection of the total magnetization, respectively.

\section{Numerical results and discussion}

In our numerical calculations we normalize the absorbed power $P_{\mathrm{FMR}}$, which is proportional to the imaginary part of the transverse magnetic susceptibility and calculate $\chi^{\prime \prime} \sim P_{\mathrm{FMR}} /\left(\mu_{0} M_{\mathrm{S}} H_{0} \omega\right)[18]$.

We have already demonstrated the sensitivity of the magnetic susceptibility to the strength of the magnetoelectric coupling $\lambda$ (Fig. 1 of Ref. [18]). Here we study the effect of the applied electric field on the spectra for symmetric MF chains of different lengths (Figs. 1 and 2).

Fig. 1 illustrates the situation for a chain with three FE- and FM-cells, each is $a_{\mathrm{FE}}=a_{\mathrm{FM}}=5[\mathrm{~nm}]$ in length. The calculation of the resonance at zero electric field involves the contribution from the exchange interaction, as inferred from eq. (4). Compared with the resonance position obtained for an ultrathin junction shown in Fig. 2 of Ref. [18], one observes a strong increase of this value resulting from an elevated frequency by a factor of five, which was dictated by the increased internal coupling within the FM system. However, the existence of the exchange interaction field entering with a negative sign into eq. (4) slightly shifts the resonance down along the field axis in Fig. 1, resulting in the resonance field of the magnitude $\mu_{0} H_{\text {res }} \approx 0.32[\mathrm{~T}]$.

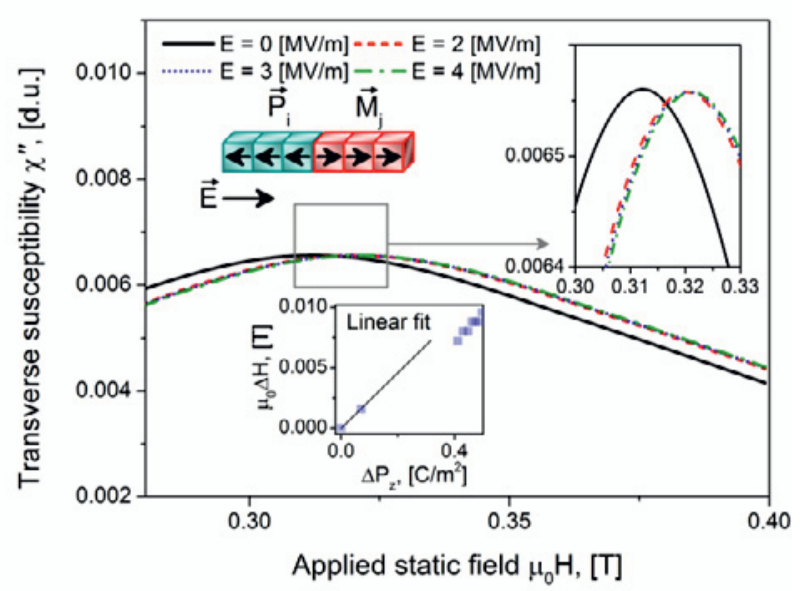

Fig. 1. FMR spectra for the MF chain of the length $N_{\mathrm{FE}}=3$ and $N_{\mathrm{FM}}=3$. The applied field frequency is $\omega /(2 \pi)=30 \cdot 10^{9}[\mathrm{~Hz}]$, FM exchange stiffness is $A=2.1 \cdot 10^{-11}[\mathrm{~J} / \mathrm{m}]$ and $\lambda=0.2[\mathrm{~s} / \mathrm{F}]$. The upper inset shows the peaks on an enlarged scale resolving the states with non-switched and switched FE layer. Other parameters are as those listed in Ref. [18].

Finite electric field (Fig. 1) gives rise to the expected modification of the resonance positions in the spectra, which is evidenced from expression (4). Especially pronounced are those states which correspond to the switched FE polarization, since the term containing $\lambda$ alters the sign in eq. (4) slightly increasing the resonance field. Although the contribution from the ME coupling to the total field decreases with enlarging the MF system, the insets of Fig. 1 demonstrate the delectability of these shifts. The lower inset of Fig. 1 demonstrates additionally the linear character of the dependence of the FMRresonance peaks on the changed polarization. The linear fitting was performed only for the initially not switched FE polarization.

In Fig. 2 we present the calculations for the MF chain of the length $N_{\mathrm{FE}}=N_{\mathrm{FM}}=10$ with unchanged 
characteristics of the external magnetic field. Fig. 2 evidences the dominant role of the exchange interaction on the $E=0$ - resonance position, since it almost returned to the values obtained for the ultrathin MF junction (Fig. 2 of Ref. [18]). It should also be noted that the absorption curve is broadened an its intensity is lower than on any other plots before. This is due to the averaging of the absorbed power, where the number of sites (thickness) enters the denominator (eq. (3)).

Remarkable is also the behaviour of the $\mu_{0} \Delta H\left(\Delta P_{\mathrm{z}}\right)$ dependence. Since the long FM chain of the magnetic moments coupled by the exchange interaction dynamically behaves similarly to the one consisting of only one FM site, the character of the resonance shifts is almost the same.

In Fig. 3 we summarize the proposed method for detection of the ME coupling strength as a function of the length of the FM part of the MF chain. Each point in this figure was obtained from a simulation of the absorption FMR-spectrum for the multiferroic chain of given length and a subsequent linear fitting of the shifts of resonance positions as a function of the altered FE polarization. All

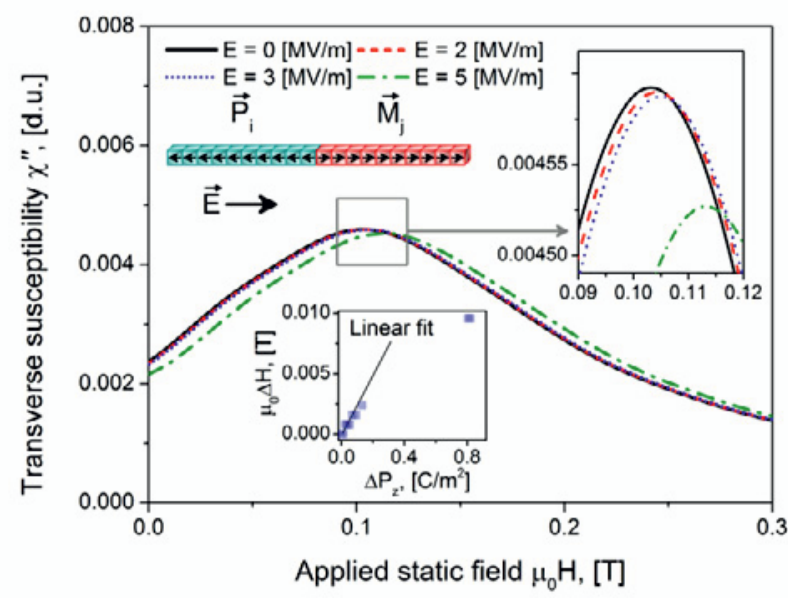

Fig. 2. FMR spectra for a long MF chain of the length $N_{\mathrm{FE}}=10$ and $N_{\mathrm{FM}}=10$. Other parameters are as those listed for Fig. 1.

fits were performed for the none-switched polarization states with the result of linear dependence, since, as mentioned in our previous study [18], the dependence of the shifts of resonances on the applied electric field showed highly nonlinear character.

In contrast to the case of an ultrathin FE layer [18], where the definition of the FE polarization was unique, the situation changes for a thicker FE layer. In the last case, although only the polarization at the interface directly influences the magnetization, we expect that experimentally the total FE polarization can be easier measured. Therefore, we decided to plot the ME coupling strength as a function of both the FE polarization directly at the interface $\left(P_{\text {Contact }}\right)$ and that averaged over the entire MF chain $\left(P_{\text {Average }}\right)$. However, our numerical results revealed almost identical behaviour for the both. This can be explained via a highly collinear polarization dynamics governed by the constant $\kappa_{\mathrm{FE}}$ in eq. (1).

In summary, we performed detailed numerical calculations of FMR-spectra of the absorbed power for
MF interfaces of different FE and FM thicknesses. For symmetric MF chains formed by FE- and FM-parts we obtained the ME coupling from fitting the shifts of resonance peaks as a function of the FE polarization modified by the applied electric field. Our numerically calculated spectra revealed a pronounced dependence of the ME coupling coefficient on the FM thickness. This dependence yielded a minimum for $\lambda$ at thicknesses of the FM around 20 [nm], or $N_{\mathrm{FM}}=4$, which can be explained due to the importance of the boundary magnetic contributions, the number of which was equal to the number of the magnetic moments inside of the FM. After approximately $N_{\mathrm{FM}}=5$ the dependence of the $\mathrm{ME}$ coupling on the FM thickness starts saturating, not reaching, however, the initially inserted value of $\lambda=0.2$ $[\mathrm{s} / \mathrm{F}]$. We attribute this to the local nature of the ME coupling effect and believe that this can be overcome by appropriate geometrical rescaling.

\section{Acknowledgements}

The authors gratefully acknowledge the support of the German Research Foundation by the Grant Numbers SU 690/1-1 and SFB 762, the grant CONACYT as Basic Science Project No. 129269 of Mexico, the Grants of the National Science Foundation of China (No. 11104123) and the National Basic Research Program of China (No. 2012CB933101).

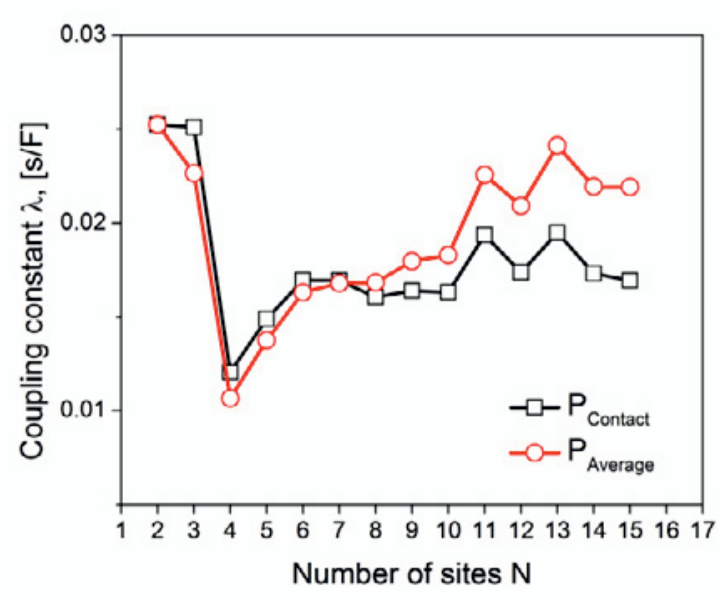

Fig. 3. Dependence of the ME coupling strength $\lambda$ obtained from the calculated FMR-spectra as a function of the length of the MF chain. The MF chain is symmetric, i.e. $N_{\mathrm{FE}}=N_{\mathrm{FM}}=N$. The calculations of $\lambda$ are performed employing eq. (7) of Ref. [18] for the polarization localized at the interface $\left(P_{\text {Contact }}\right)$ and for the polarization $\left(P_{\text {Average }}\right)$ averaged over the entire chain.

\section{References}

1. M. Fiebig, J. Phys. D: Appl. Phys. 38, R123 (2005).

2. W. Eerenstein, N.D. Mathur, and J.F. Scott, Nature 442, 759 (2006).

3. R. Ramesh and N.A. Spaldin, Nature Mater. 6, 21 (2007). 
4. M. Bibes and A. Barthélémy, Nature Mater. 7, 425 (2008).

5. S.-W. Cheong, M. Mostovoy, Nature Mater. 6, 13 (2007).

6. C.A.F. Vaz, J. Hoffman, C.H. Ahn, and R. Ramesh, Adv. Mater. 22, 2900 (2010).

7. G. Lawes and G. Srinivasan, J. Phys. D: Appl. Phys. 44, 243001 (2011).

8. C.-G. Duan, S.S. Jaswal, and E.Y. Tsymbal, Phys. Rev. Lett. 97, 047201 (2006).

9. T. Cai, S. Ju, J. Lee, N. Sai, A.A. Demkov, Q. Niu, Z. Li, J. Shi, and E. Wang, Phys. Rev. B 80, 140415(R) (2009).

10. M. Fechner, I.V. Maznichenko, S. Ostanin, A. Ernst, J. Henk, and I. Mertig, Phys. Stat. Solidi B 247, 1600 (2010).

11. S. Valencia, A. Crossous, L. Bocher, V. Garcia, X. Moya, R.O. Cherifi, C. Deranlot, K. Bouzehouane, S. Fusil, A. Zobelli, A. Gloter, N.D. Mathur, A. Gaupp, R. Arudan, F. Radu, A. Barthélémy, and M. Bibes, Nature Mater. 10, 753 (2011).

12. H.L. Meyerheim, F. Klimenta, A. Ernst, K. Mohseni, S. Ostanin, M. Fechner, S. Parihar, I.V. Maznichenko, I. Mertig, and J. Kirschner, Phys. Rev. Lett. 106, 087203 (2011).

13. W.H. Meiklejohn and C.P. Bean, Phys. Rev. 105, 904 (1957).

14. J. Nogués, I.K. Schuller, J. Magn. Magn. Mater. 192, 203 (1999).

15. J.H. Griffiths, Nature 158, 670 (1946).

16. E.K. Zavoiskii, J. Phys. U.S.S.R. 10, 170 (1946).

17. M. Farle, Rep. Prog. Phys. 61, 755 (1998).

18. A. Sukhov, P.P. Horley, C.-L. Jia, and J. Berakdar, J. Appl. Phys. 113, 013908 (2013) and references therein.

19. A. Sukhov, C.-L. Jia, P.P. Horley, and J. Berakdar, J. Phys.: Condens. Matter 22, 352201 (2010).

20. P.P. Horley, A. Sukhov, C.-L. Jia, E. Martínez, and J. Berakdar, Phys. Rev. B 85, 054401 (2012). 